\title{
On Finite Subnormal Cayley Graphs
}

\author{
Shu Jiao Song* \\ School of Mathematics and Information Science \\ Yantai University, China. \\ shujiao.song@hotmail.com
}

Submitted: Oct 10, 2020; Accepted: Aug 8, 2021; Published: Aug 27, 2021

(C) Shu Jiao Song. Released under the CC BY-ND license (International 4.0).

\begin{abstract}
In this paper we introduce and study a type of Cayley graph - subnormal Cayley graph. We prove that a subnormal 2-arc transitive Cayley graph is a normal Cayley graph or a normal cover of a complete bipartite graph $\mathbf{K}_{p^{d}, p^{d}}$ with $p$ prime. Then we obtain a generic method for constructing half-symmetric (namely edge transitive but not arc transitive) Cayley graphs.
\end{abstract}

Mathematics Subject Classifications: 05C25, 20B05

\section{Introduction}

For a finite group $G$ and a subset $S \subset G$, the Cayley digraph $\Gamma=\operatorname{Cay}(G, S)$ is the digraph with vertices being the elements of $G$ such that $x, y \in G$ are adjacent if and only if $y x^{-1} \in S$. If $S=S^{-1}=\left\{s^{-1} \mid s \in S\right\}$, then the adjacency is symmetric and thus Cay $(G, S)$ may be viewed as an (undirected) graph, that is, a Cayley graph. Let

$$
\widehat{G}=\{\widehat{g}: x \mapsto x g \text { for all } x \in G \mid g \in G\} .
$$

Then $\widehat{G} \leqslant$ Aut $\Gamma$, and $\widehat{G}$ acts regularly on the vertex set $G$, so $\Gamma$ is vertex-transitive.

A Cayley graph $\Gamma=\operatorname{Cay}(G, S)$ is called normal if $\widehat{G}$ is normal in Aut $\Gamma$. The class of normal Cayley graphs have nice properties and play an important role in studying Cayley graphs, see $[5,6,10,11,16,19]$ and references therein. However, there are various interesting classes of Cayley graphs which are not normal.

Here we generalize the concept of normal Cayley graphs. For a group $Y$, a subgroup $X$ of $Y$ is called subnormal if there exists a sequence of subgroups $X_{0}, X_{1}, \ldots, X_{l}$ of $Y$ such that $X=X_{0} \triangleleft X_{1} \triangleleft \cdots \triangleleft X_{l}=Y$; denoted by $X \triangleleft \triangleleft Y$. A Cayley graph $\Gamma=\operatorname{Cay}(G, S)$ is called subnormal if $\widehat{G}$ is subnormal in Aut $\Gamma$, and more generally, $\Gamma$ is called $Y$-subnormal

\footnotetext{
*Supported by NSFC(No.61771019) and NSFS (No.ZR2020MA044).
} 
if $\widehat{G}$ is subnormal in $Y$, where $\widehat{G} \leqslant Y \leqslant$ Aut $\Gamma$. In the case where $\widehat{G} \triangleleft \triangleleft Y$ and $\Gamma$ is $Y$-edge transitive, $Y$-arc transitive or $(Y, 2)$-arc transitive, $\Gamma$ is called subnormal edge transitive, subnormal arc transitive, or subnormal 2-arc transitive, respectively. (A graph $\Gamma$ is called $(Y, 2)$-arc transitive if $Y \leqslant \Gamma$ is transitive on the set of 2-arcs of $\Gamma$.) This paper initiates to study the class of subnormal Cayley graphs.

Typical examples of subnormal Cayley graphs include generalized orbital graphs of quasiprimitive permutation groups of simple diagonal type or compound diagonal type, refer to [15]. The class of 2-arc transitive graphs is one of the central objects in algebraic graph theory, see $[10,15]$ for references. It is shown in [10] that there are only finite many 'basic' 2-arc transitive Cayley graphs of given valency which are not normal. Here we show that almost all subnormal 2-arc transitive Cayley graphs are normal.

Let $\Gamma$ be a $(Y, 2)$-arc transitive graph with vertex set $V$. Let $N$ be a normal subgroup of $Y$ which has at least three orbits on $V$. Let $\mathcal{B}$ be the set of $N$-orbits on $V$. The normal quotient $\Gamma_{N}$ of $\Gamma$ induced by $N$ is the graph with vertex set $\mathcal{B}$ such that $B, B^{\prime} \in \mathcal{B}$ are adjacent if and only if some vertex $u \in B$ is adjacent in $\Gamma$ to some vertex $v \in B^{\prime}$. Let $K$ be the kernel of $Y$ acting on $\mathcal{B}$. Then $K_{\alpha}=1$ as $K_{\alpha} \triangleleft Y_{\alpha}$ and $Y_{\alpha}$ is 2-transitive on $\Gamma(\alpha)$. Thus $\Gamma_{N}$ is $(Y / N, 2)$-arc transitive of valency equal the valency of $\Gamma$, and so $\Gamma$ is a normal cover of $\Gamma_{N}$, that is, $\{u, v\}$ is an edge in $\Gamma$, then the induced subgraph $\left[u^{N}, v^{N}\right]$ is a pefect matching.

Theorem 1. Let $\Gamma=\operatorname{Cay}(G, S)$ be connected and undirected. Assume that $Y \leqslant \operatorname{Aut} \Gamma$ is such that $\widehat{G} \triangleleft \triangleleft Y$ and $\Gamma$ is $(Y, 2)$-arc transitive. Then either $\widehat{G} \triangleleft Y$, or $\widehat{G} \triangleleft X \triangleleft Y$ and $\Gamma$ is a normal cover of the complete bipartite graph $\mathbf{K}_{p^{d}, p^{d}}$, where $p$ is an odd prime.

This theorem is proved in Section 3. The next theorem is a by-product for proving Theorem 1, which extends a classical result for primitive permutation groups, that is, [3, Theorem 3.2C] and [18, Theorems 18.4 and 18.5], to the general transitive permutation groups. Some special cases of this result have been obtained and used in the study of symmetrical graphs, see for example, [9, Lemma 2.1] and [4, Lemma 2.1].

Edge transitive graphs are divided into three disjoint classes: symmetric (arc transitive); semi-symmetric (vertex intransitive); half-symmetric (vertex transitive but not arc transitive). We remark that in the literature, half-symmetric graphs were called halftransitive graphs. However, since 'half-transitive' is a classical concept for permutation groups and often occurs in the area of transitive graphs, we would prefer to call them 'half-symmetric' instead of 'half-transitive'.

Constructing and characterizing half-symmetric graphs is an active topic in symmetrical graph theory which has received considerable attention, see for example $[11,12,14,17]$. The following result provides a generic method for constructing half-symmetric graphs as subnormal Cayley graphs, which is proved in Section 4.

Theorem 2. Let $T$ be a finite simple group containing an element $t$ which is not conjugate in $\operatorname{Aut}(T)$ to $t^{-1}$. Let $G=T^{l}$ with $l \geqslant 2$, and let

$$
R=\left\{\left(t^{x}, 1, \ldots, 1\right),\left(1, t^{x}, \ldots, 1\right), \ldots,\left(1,1, \ldots, t^{x}\right),\left(t^{x}, t^{x}, \ldots, t^{x}\right) \mid x \in T\right\} .
$$

Then $\operatorname{Cay}\left(G, R \cup R^{-1}\right)$ is subnormal and half-symmetric. 
Many finite simple groups $T$ contain elements which are not conjugate in $\operatorname{Aut}(T)$ to their inverses. Here is an example. Let $T=\operatorname{Sz}(q)$ with $q=2^{2 e+1} \geqslant 8$, and let $t$ be an element of $T$ of order 4 . Then $t$ is not conjugate in Aut $(T)$ to $t^{-1}$.

\section{Proof of Theorem 1}

Let $\Gamma=(V, E)$ be a digraph. For $v \in V$, let $\Gamma(v)=\{w \in V \mid(v, w)$ is an arc of $\Gamma\}$. Let $G_{v}^{[1]}$ be the kernel of $G_{v}$ acting on $\Gamma(v)$. Then $G_{v}^{[1]}$ is normal in $G_{v}$. Let $\Gamma_{0,1, \cdots, i}(v)=\{w \mid$ the distance between $v$ and $d$ are not larger than $i$ in $\Gamma$ \}. We first prove a simple lemma about the vertex stabilisers of vertex transitive graphs.

Lemma 3. Let $\Gamma$ be a connected $G$-vertex transitive digraph. Then for a vertex $v$ and a normal subgroup $N \triangleleft G$, if $N_{v}^{\Gamma(v)}$ is semiregular, then $N_{v} \cong N_{v}^{\Gamma(v)}$ is faithful.

Proof. Suppose that $N_{v}^{\Gamma(v)}$ is semiregular for a $v$. Since $N \triangleleft G$, for any $w \in \Gamma$, there is an element $g \in G$ such that $w=v^{g}$. Thus $N_{v^{g}}=N_{v}^{g}$ and $N_{w}^{\Gamma(w)}$ is semiregular. For the contrary, suppose that there exists an $x \in N$ such that $x$ fixes pointwesely $\Gamma_{0,1}(v)$. Let $i \geqslant 1$ be the maximal integer such that $x$ fixes $\mathrm{G}_{0,1, \cdots, i}(v)$ but moves a vertex $w^{\prime} \in \Gamma_{i+1}(v)$. Let $v^{\prime} \in \Gamma_{i-1}(v), w \in \Gamma_{i}(v)$ and $w^{\prime} \in \Gamma_{i+1}(v)$ such that $\left(v^{\prime}, w, w^{\prime}\right)$ is a 2-arc. Then $x$ fixes $v^{\prime}, w$, and moves $w^{\prime}$. Thus $x \in G_{w v^{\prime}}$ and acts non-trivially on $\Gamma_{1}(w)$. So $N_{w}^{\Gamma(w)}$ is not semiregular, a contradiction.

For a group $X$ and a core free subgroup $H \leqslant X$, denote by $[X: H]$ the set of right cosets of $H$ in $X$, that is

$$
[X: H]=\{H x \mid x \in X\} .
$$

For any subset $S \subset X$, define the coset graph of $X$ with respect to $H$ and $S$ to be the digraph $\Gamma$ with vertex set $[X: H]$ and such that two vertices $H x, H y \in V$ are adjacent, written as $H x \sim H y$, if and only if $y x^{-1} \in H S H$; denoted by $\Gamma=\operatorname{Cos}(X, H, H S H)$. Then $X \leqslant$ Aut $\Gamma$, and $\Gamma$ is $X$-vertex transitive. For convenience, write $H\{g\} H=H g H$, where $g \in X$. The following properties are known and easy to prove.

Lemma 4. Let $X$ be a group, $H$ a core free subgroup, and $g \in X$. Then

(i) $\operatorname{Cos}(X, H, H g H)$ is connected if and only if $\langle H, g\rangle=X$;

(ii) $\operatorname{Cos}(X, H, H g H)$ is $X$-edge transitive;

(iii) $\Gamma=\operatorname{Cos}\left(X, H, H\left\{g, g^{-1}\right\} H\right)$ is undirected and $X$-edge transitive; further, $\Gamma$ is $X$ arc transitive if and only if $\mathrm{HgH}=\mathrm{Hg}^{-1} \mathrm{H}$.

Let $\operatorname{Aut}(X, H)=\left\langle\sigma \in \operatorname{Aut}(X) \mid H^{\sigma}=H\right\rangle$. Then an element $\sigma \in \operatorname{Aut}(X, H)$ acts on $[X: H]$ by $(H x)^{\sigma}=H x^{\sigma}$. Let $\sigma \in \operatorname{Aut}(X, H)$ be such that $(H g H)^{\sigma}=H g H$. Then for any two vertices $H x, H y$, we have

$$
\begin{aligned}
H x \sim H y & \Leftrightarrow y x^{-1} \in H g H \\
& \Leftrightarrow y^{\sigma}\left(x^{\sigma}\right)^{-1}=\left(y x^{-1}\right)^{\sigma} \in(H g H)^{\sigma}=H g H \\
& \Leftrightarrow H x^{\sigma} \sim H y^{\sigma}
\end{aligned}
$$


Thus $\sigma$ maps all edges to edges, and so $\sigma$ induces an automorphism of $\Gamma$.

Lemma 5. Let $\Gamma=\operatorname{Cos}(X, H, H g H)$, and $\sigma \in \operatorname{Aut}(X, H)$. If $(H g H)^{\sigma}=H g H$, then $\sigma$ induces an automorphism of $\Gamma$.

For a group $G$, the symmetric group $\operatorname{Sym}(G)$ contains two regular subgroups $\widehat{G}$ and $\check{G}$, where

$$
\check{G}=\left\{\check{g}: x \mapsto g^{-1} x \text { for all } x \in G \mid g \in G\right\},
$$

consisting of left multiplications of elements $g \in G$ and $\hat{G}$ with

$$
\hat{G}=\{\hat{g}: x \mapsto x g \text { for all } x \in G \mid g \in G\},
$$

consisting of right multiplications of elements $g \in G$. Then by [7], $\mathbf{N}_{\operatorname{Sym}(G)}(\widehat{G})=\widehat{G} \rtimes$ Aut $(G)$, the holomorph of $G$, and $\widehat{G} \mathbf{C}_{\operatorname{Sym}(G)}(\widehat{G})=\widehat{G} \circ \check{G}=\widehat{G} \rtimes \operatorname{lnn}(G)$.

For a subset $S \subset G$, let

$$
\operatorname{Aut}(G, S)=\left\{\sigma \in \operatorname{Aut}(G) \mid S^{\sigma}=S\right\} .
$$

Then $\operatorname{Aut}(G, S) \leqslant \operatorname{Aut}(G) \leqslant \operatorname{Sym}(G)$, and as subgroups of $\operatorname{Sym}(G)$, it is easily shown that $\operatorname{Aut}(G, S)$ normalizes $\widehat{G}$. Moreover, for the Cayley graph $\Gamma=\operatorname{Cay}(G, S)$, by $[7$, Lemma 2.1], we have

$$
\mathbf{N}_{\text {Aut } \Gamma}(\widehat{G})=\widehat{G} \rtimes \operatorname{Aut}(G, S) .
$$

The subgroup Aut $(G, S)$ plays an important role in the study of Cayley graphs. Assume that If $\widehat{G} \triangleleft X \leqslant A u t \Gamma$. Then $X_{\alpha} \leqslant \operatorname{Aut}(G, S)$ where $\alpha$ is a vertex of $\Gamma$. A special type of normal Cayley graph satisfies $X_{\alpha} \geqslant \operatorname{lnn}(G, S)$, in this case, we call $\Gamma$ a holomorph Cayley graph.

Suppose $\Gamma=\operatorname{Cay}(G, S)$ is a holomorph graph with $H=\widehat{G} \circ \check{G}=\widehat{G} \rtimes \operatorname{lnn}(G)$. Let $\beta \in \Gamma(\alpha)=S$, let $g \in H_{\alpha \beta}$, then $\beta^{h}=\beta$, that is $h \in \mathbf{C}_{G}(\beta)$. On the contrary, if $h \in \mathbf{C}_{G}(\beta)$, then $\beta^{h}=\beta$, so $h \in H_{\alpha \beta}$. Thus $H_{\alpha \beta}=\mathbf{C}_{G}(\beta)$. Thus the following lemma holds.

Lemma 6. Suppose $\Gamma=\operatorname{Cay}(G, S)$ is a holomorph with $H=\widehat{G} \circ \check{G}$. Then $H_{\alpha \beta}=\mathbf{C}_{G}(\beta)$.

The next lemma shows that, for a prime $p$ and an integer $d$, a complete bipartite graph $\mathbf{K}_{p^{d}, p^{d}}$ is a 2-arc transitive subnormal Cayley graph.

Lemma 7. Let $\Gamma=\mathbf{K}_{p^{d}, p^{d}}$, where $p$ is an odd prime and $d \geqslant 1$. Then $\Gamma \cong \operatorname{Cay}(G, S)$, where $G \cong \mathbb{Z}_{p}^{d} \rtimes \mathbb{Z}_{2}$ and $S$ consists of all involutions of $G$, and there exist subgroups $X, Y<$ Aut $\Gamma$ such that $\widehat{G} \triangleleft X \triangleleft Y<\operatorname{Aut} \Gamma, X=\widehat{G} \rtimes \operatorname{Aut}(G)$, and $Y / X \cong \mathbb{Z}_{2}$.

Proof. Let $G=N \rtimes\langle z\rangle \cong \mathbb{Z}_{p}^{d} \rtimes \mathbb{Z}_{2}$, where $p$ is an odd prime and $z$ reverses every element of $N$, that is, for each element $x \in N, x^{z}=x^{-1}$. Let $S=G \backslash N$, and let $\Gamma=\operatorname{Cay}(G, S)$. Then $S$ consists of all involutions of $G$. Let $V_{1}$ be the vertex set corresponding to the elements in $N, V_{2}$ be the vertex set corresponding to the elements in $G \backslash N$. Then each vertex in $V_{1}$ is adjcent to all vertices in $V_{2}$ and each vertex in $V_{2}$ is adjcent to all vertices 
in $V_{1}$ as well. So $\Gamma \cong \mathbf{K}_{p^{d}, p^{d}}$. Thus, Aut $\Gamma \cong \S_{p^{d}} 2 \S_{2}$. Further, $\operatorname{Aut}(G, S)=\operatorname{Aut}(G) \cong$ $\operatorname{AGL}(d, p)=\mathbb{Z}_{p}^{d} \rtimes \operatorname{GL}(d, p)$, and Aut $(G, S)$ acts 2-transitively on $S$.

Let $X=\mathbf{N}_{\text {Aut } \Gamma}(\widehat{G})$, and let $C=\widehat{G} \mathbf{C}_{\text {Aut } \Gamma}(\widehat{G})$. Then $X=\widehat{G} \rtimes \operatorname{Aut}(G, S)=\widehat{G} \rtimes \operatorname{Aut}(G)$, and $C=\widehat{G} \times \check{G}$. Thus $\Gamma$ is $(X, 2)$-arc transitive and $C$-arc transitive. Let $v$ be the vertex of $\Gamma$ corresponding to the identity of $G$. Then $C_{v}=\{(\widehat{g}, \check{g}) \mid g \in G\} \cong G$. Let $\Gamma^{\prime}=\operatorname{Cos}\left(C, C_{v}, C_{v}(\widehat{z}, 1) C_{v}\right)$ and $\phi$ a map from vetices of $\Gamma$ to vertices of $\Gamma^{\prime}$ such that for any vertex $C_{v} x \in V \Gamma^{\prime}$ and $x \in V \Gamma, \phi: C_{v} x \mapsto x$. Then $\phi$ is an isomorphism of $\Gamma$ to $\Gamma^{\prime}$. Thus $\Gamma \cong \operatorname{Cos}\left(C, C_{v}, C_{v}(\widehat{z}, 1) C_{v}\right)$.

We label Aut $(\widehat{G})=\{\widehat{x} \mid x \in \operatorname{Aut}(G)\}$, and $\operatorname{Aut}(\check{G})=\{\check{x} \mid x \in \operatorname{Aut}(G)\}$. Then $\operatorname{Aut}(C)=\operatorname{Aut}(\widehat{G} \times \check{G})=(\operatorname{Aut}(\widehat{G}) \times \operatorname{Aut}(\check{G})) \cdot\langle\tau\rangle$, where $\tau:(\widehat{x}, \check{y}) \mapsto(\widehat{y}, \check{x})$ for all $(\widehat{x}, \check{y}) \in$ $\operatorname{Aut}(\widehat{G}) \times \operatorname{Aut}(\check{G})$. Let $(\widehat{x}, \check{y}) \in \operatorname{Aut}(C)$ normalize $C_{v}=\{(\widehat{g}, \check{g}) \mid g \in G\}$. Then $\left(\widehat{g}^{\widehat{x}}, \check{g}^{\check{y}}\right) \in C_{v}$ for any $g \in G$. Thus $g^{y x^{-1}}=g$ for any $g \in G$, that is $y x^{-1} \in \mathbb{Z}(G)=1$. Hence $x=y$ and Aut $\left(C, C_{v}\right)=\langle(\widehat{x}, \check{x}) \mid x \in \operatorname{Aut}(G)\rangle \times\langle\tau\rangle$. Since $C \triangleleft X$ and $\mathbf{C}_{X}(C)=1$, it follows that $X \leqslant \operatorname{Aut}(C)$. Further, $C_{v} \triangleleft X_{v} \leqslant \operatorname{Aut}\left(C, C_{v}\right)$, and it follows that Aut $\left(C, C_{v}\right)=X_{v} \times\langle\tau\rangle$. Noticing that $(\widehat{z}, \check{z}) \in C_{v}$ and $\check{z}$ is an involution, we have

$$
\left(C_{v}(\widehat{z}, 1) C_{v}\right)^{\tau}=C_{v}(\widehat{z}, 1)^{\tau} C_{v}=C_{v}(1, \check{z}) C_{v}=C_{v}(1, \check{z})(\widehat{z}, \check{z}) C_{v}=C_{v}(\widehat{z}, 1) C_{v} .
$$

By Lemma 5, $\tau \in \operatorname{Aut} \Gamma$ and $\operatorname{Aut}\left(C, C_{v}\right)<\operatorname{Aut} \Gamma$. Now $Y:=C \operatorname{Aut}\left(C, C_{v}\right)$ is such that $|Y: X|=2$. We obtain that $\widehat{G} \triangleleft X \triangleleft Y<$ Aut $\Gamma$. Since $\tau \in Y$ does not normalizes $\widehat{G}, \widehat{G}$ is not normal in $Y$.

Therefore, as $\Gamma$ is $(Y, 2)$-arc transitive, $\mathbf{K}_{p^{d}, p^{d}}$ is a 2 -arc transitive subnormal Cayley graph.

The following is a property regarding 2-transitive permutation groups, which is obtained by inspecting of the classification of 2-transitive permutation groups, refer to [3].

Lemma 8. Let $X$ be a 2-transitive permutation group on $\Omega$. Then the socle of $X$ is either a regular elementary abelian p-group, or a nonregualr nonabelian simple group.

Furter, assume that $N \triangleleft \triangleleft X$ is imprimitive on $\Omega$. Then $X$ is affine with $\operatorname{soc}(X)=\mathbb{Z}_{p}^{e}$, where $p$ is a prime and $e \geqslant 1$, and further, the following hold:

(i) Either $N \leqslant \operatorname{soc}(X)$, or $\mathbb{Z}_{p}^{e} \cdot \mathbb{Z}_{b} \cong N \triangleleft X$ and $N$ is a Frobenius group, where $b$ divides $p^{e^{\prime}}-1$ and $e^{\prime}$ is a proper divisor of $e$.

(ii) $X_{\omega}$ has no non-trivial normal subgroup of p-power order for $\omega \in \Omega$.

Proof. By the classification of 2-transitive groups, we know that $X$ is either almost simple or affine.

(1) Suppose that $X$ is almost simple, that is $T \leqslant X \leqslant$ Aut $(T)$, where $T \cong \operatorname{lnn}(T)$ is nonabelian simple. For any $1<N \triangleleft X$, suppose $T \nsubseteq N$. Then from $T \cap N \triangleleft T$ we have $T \cap N=1$ and then $T \times N \leqslant X \leqslant \operatorname{Aut}(T)$, which implies $N=1$, a contradiction. So $T \leqslant N$, that means that $N$ is also almost simple. Repeating this process, we know that $T \leqslant N$ and so $N \triangleleft X$, and $N$ is primitive. 
(2) Suppose that $X$ is affine with the socle $S=\mathbb{Z}_{p}^{e}$. Set $n=p^{e}$. Then $X=S \rtimes H$, where $H$ is a transitive subgroup of $\mathrm{GL}(e, p)$ on nonzero vectors. By [8], $X_{\omega} \cong H$ has no non-trivial normal subgroup of p-power order for $\omega \in \Omega$ as in item (ii).

Clearly, every subgroup of $S$ is subnormal in $X$. So we assume that $N \varsubsetneqq S$ below. For any $1 \neq N \triangleleft X$, suppose that $S \cap N=1$. Then $S N=S \times N \leqslant S \rtimes H$ which implies $N \leqslant S$, a contradiction. So $N \cap S \neq 1$. Since $H$ is transitive on nonzero vectors, we have $(N \cap S)^{H}=S$, which implies $S \leqslant N$. Then $N / S \triangleleft \bar{X}=X / S=\bar{H}$. By Lemma 5.1 in [8], we have if $N$ is imprimitive, then $N / S \cong \mathbb{Z}_{b}$, as in item (i).

This has an application to 2-arc transitive graphs.

Lemma 9. Let $\Gamma$ be a $(Y, 2)$-arc transitive graph, and let $H$ be a subnormal subgroup of $Y$ which is vertex transitive on $\Gamma$. Then either $H_{v}^{\Gamma(v)}$ is center free and $\Gamma$ is $H$-arc transitive, or $H_{v}$ is abelian and acts faithfully and semiregularly on $\Gamma(v)$.

Proof. Since $H \triangleleft \triangleleft Y$, we have that $H_{v} \triangleleft \triangleleft Y_{v}$, and $H_{v}^{\Gamma(v)} \triangleleft \triangleleft Y_{v}^{\Gamma(v)}$ and $Y_{v}^{\Gamma(v)}$ is a 2-transitive permutation group. If $H_{v}^{\Gamma(v)}$ is primitive, then $\Gamma$ is $H$-arc transitive and $H_{v}^{\Gamma(v)} \geqslant \operatorname{soc}\left(Y_{v}^{\Gamma(v)}\right)$ is center free by Lemma 8 .

Now suppose $H_{v}^{\Gamma(v)}$ is imprimitive. Since $Y_{v}^{\Gamma(v)}$ is a 2-transitive permutation group, it follows from Lemma 8 that either $H_{v}^{\Gamma(v)} \leqslant \operatorname{soc}\left(Y_{v}^{\Gamma(v)}\right) \cong \mathbb{Z}_{p}^{e}$, where $p$ is a prime and $e \geqslant 1$ or $\operatorname{soc}\left(Y_{v}^{\Gamma(v)}\right)=\mathbb{Z}_{p}^{e} \leqslant H_{v}^{\Gamma(v)}=\mathbb{Z}_{p}^{e} \cdot \mathbb{Z}_{b}$ and $H_{v}^{\Gamma(v)}$ is center free.

For the former, since $\operatorname{soc}\left(Y_{v}^{\Gamma(v)}\right)$ is regular, $H_{v}^{\Gamma^{(v)}}$ is semiregular. By Theorem 3, $H_{v} \cong H_{v}^{\Gamma(v)}$ is faithful and abelian.

For the latter, since $Y_{v}^{\Gamma(v)}$ is 2-transitive, we have that $H_{v}^{\Gamma(v)} \geqslant \operatorname{soc}\left(Y_{v}^{\Gamma(v)}\right)$ is transitive, and hence $\Gamma$ is $H$-arc transitive.

To prove Theorem 1, we need the next property on permutation groups.

Lemma 10. Let $G_{1}, G_{2}<\operatorname{Sym}(\Omega)$ be regular which normalizes each other. If $G_{1} /\left(G_{1} \cap\right.$ $\left.G_{2}\right)$ is abelian, then $G_{1}=G_{2}$.

Proof. Let $X=G_{1} G_{2}$, and $C=G_{1} \cap G_{2}$. Then $C$ is semiregular on $\Omega$, and $C \triangleleft X$. Let $\bar{G}_{1}=G_{1} / C, \bar{G}_{2}=G_{2} / C$, and $\bar{X}=X / C$. Let $\Omega_{C}$ be the set of $C$-orbits on $\Omega$. Then both $\bar{G}_{1}$ and $\bar{G}_{2}$ are regular on $\Omega_{C}$ as $G_{1}, G_{2}$ are both regular on $\Omega$.

Suppose that $G_{1} \neq G_{2}$. Then $\bar{G}_{i} \neq 1$, and $\bar{X}=\bar{G}_{1} \times \bar{G}_{2}$. In particular, $\bar{G}_{2} \leqslant$ $\mathrm{C}_{\mathrm{Sym}\left(\Omega_{C}\right)}\left(\bar{G}_{1}\right)$. If $\bar{G}_{1}$ is abelian, then $\bar{G}_{2} \leqslant \mathbf{C}_{\mathrm{Sym}\left(\Omega_{C}\right)}\left(\bar{G}_{1}\right)=\bar{G}_{1}$. Thus $\bar{G}_{2}=\bar{G}_{1}$, and so $G_{1}=G_{2}$, which is a contradiction.

Now we are ready to prove Theorem 1.

Proof of Theorem 1: Let $\Gamma=\operatorname{Cay}(G, S)$ be a $(Y, 2)$ subnormal arc transitive graph with vertex set $V$. Then $G$ is regular on $V$.

If $G \triangleleft Y$ then the theorem holds. Now we supppose $G \Varangle Y$. Then $\mathbf{N}_{\text {Aut } \Gamma}(G)<Y$. Let $X$ be the maximal subnormal subgroup of $Y$ contained in $\mathbf{N}_{\text {Aut } \Gamma}(G)$, we have $G \triangleleft X \triangleleft \triangleleft Y$. If $X \triangleleft Y$ then $\mathbf{N}_{Y}(X)=Y>\mathbf{N}_{\text {Aut } \Gamma}(G)$, otherwise there is a group $K>X$ such 
that $X \triangleleft K \triangleleft \triangleleft Y$, so $\mathbf{N}_{Y}(X) \geqslant K$ with $K \cap \mathbf{N}_{\text {Aut } \Gamma}(G)=X$ as $X$ is maximal. Thus $\mathbf{N}_{Y}(X) \neq \mathbf{N}_{\text {Aut } \Gamma}(G)$. Since $G<X<\mathbf{N}_{Y}(X) \leqslant Y$, any element $y \in \mathbf{N}_{Y}(X) \backslash \mathbf{N}_{Y}(G)$ is such that $G^{y} \neq G$ and $X^{y}=X$.

Let $C=G \cap G^{y}$. Then for any $x \in X$, we have $G^{x}=G,\left(G^{y}\right)^{x}=G^{y x}=G^{x^{\prime} y}=G^{y}$ for some $x^{\prime} \in X$. Thus $C^{x}=C$ and $C, G, G^{y} \triangleleft X$; in particular, $G$ and $G^{y}$ normalizes each other. Let $\bar{G}=G / C$ and $\bar{G}^{y}=G^{y} / C$, let $V_{C}$ be the set of $C$-orbits on $V$. By Lemma $10, \bar{G}$ is not abelian as $G \neq G^{y}$. Since $G, G^{y}$ are both regular on $V$, the subgroup $C$ is semiregular on $V$, and $\bar{G}, \bar{G}^{y}$ are both regular on $V_{C}$. Further, $\bar{G}^{y} \leqslant \mathbf{C}_{\operatorname{Sym}\left(V_{C}\right)}(\bar{G})$.

Let $H=G G^{y}$. Then $H \triangleleft X$. Let $\bar{H}=H / C$, and $\bar{X}=X / C$. Then $\bar{G} \times \bar{G}^{y}=\bar{H} \triangleleft \bar{X}$. Let $v$ be a vertex of $\Gamma$. Then $H=G: H_{v}=G^{y}: H_{v}$, and $H_{v} \cong H / G^{y} \cong G / C=\bar{G}$. Further, since $G<H \triangleleft X \triangleleft \triangleleft Y$, we have $1 \neq H_{v} \triangleleft X_{v} \triangleleft \triangleleft Y_{v}$, and $1 \neq H_{v}^{\Gamma(v)} \triangleleft X_{v}^{\Gamma(v)} \triangleleft \triangleleft Y_{v}^{\Gamma(v)}$. By Lemma 8, we conclude that either $\operatorname{soc}\left(Y_{v}^{\Gamma(v)}\right) \leqslant H_{v}^{\Gamma(v)}$, or $Y_{v}^{\Gamma(v)}$ is affine with socle isomorphic to $\mathbb{Z}_{p}^{d}, H_{v}^{\Gamma(v)}<\operatorname{soc}\left(Y_{v}^{\Gamma(v)}\right) \cong \mathbb{Z}_{p}^{d}$, and $H_{v}^{\Gamma(v)}$ is semiregular.

Let $\alpha$ be the vertex of $\Gamma_{C}$ containing $v$, that is, $\alpha=v^{C}$. Then the stabilizer $\bar{H}_{\alpha}$ is isomorphic to $\bar{G}$ as $\bar{G} \times \bar{G}^{y}=\bar{H}=\bar{G}: \bar{H}_{\alpha}=\bar{G}^{y}: \bar{H}_{\alpha}$. On the other hand, $\bar{H}_{\alpha}$ is isomorphic to a factor group of $H_{v}$, that is, $\bar{H}_{\alpha} \cong H_{v} C / C \cong H_{v} /\left(H_{v} \cap C\right)$.

Suppose that $H_{v}$ is abelian. Then the factor group $\bar{H}_{\alpha} \cong H_{v} /\left(H_{v} \cap C\right)$ is abelian. Since $\bar{G} \cong \bar{G}^{y} \cong \bar{H}_{\alpha}$, we conclude that $G$ is abelian by Lemma 10 , which is a contradiction. Thus, $H_{v}$ is not abelian. By Lemmas 8 and 9 , either $Y_{v}^{\Gamma(v)}$ is almost simple, or $H_{v}^{\Gamma(v)}=$ $\mathbb{Z}_{p}^{d}: H_{o}$ is a Frobenius group. In particular, $H_{v}$ is transitive on $\Gamma(v)$, and $\Gamma$ is $H$-arc transitive.

Since $\bar{G} \times \bar{G}^{y}=\bar{H} \leqslant \operatorname{Aut}\left(\Gamma_{C}\right)$, and $\bar{G}$ is regular on $\Gamma_{C}$, we have $\Gamma_{C}$ is a holomorph graph Cay $(\bar{G}, S)$. So $\bar{H}_{\alpha}^{\Gamma_{C}(\alpha)} \cong \bar{H}_{\alpha}=\operatorname{lnn}(\bar{G}, S)$. Suppose that $Y_{v}^{\Gamma(v)}$ is almost simple. Then $\operatorname{soc}\left(Y_{v}^{\Gamma(v)}\right) \leqslant H_{v}^{\Gamma(v)} \leqslant Y_{v}^{\Gamma(v)}$. Since $Y_{v}^{\Gamma(v)}$ is 2-transitive, by the classification of 2-transitive almost simple groups, see [2], either $H_{v}^{\Gamma(v)}$ is 2-transitive, or $|\Gamma(v)|=28$, $H_{v}^{\Gamma(v)} \cong \operatorname{PSL}(2,8)$ and $Y_{v}^{\Gamma(v)} \cong \operatorname{P\Gamma L}(2,8)$. For the former, the graph $\Gamma_{C}$ is a holomorph 2 -arc transitive graph, which is not possible, see [11, Theorem 1.3]. For the latter, since $\left|\Gamma_{C}(\alpha)\right|=|\Gamma(v)|=28$, we have $\bar{H}_{\alpha}=\mathrm{D}_{18}$ which have index 28 in $\bar{H}_{\alpha}=\operatorname{PSL}(2,8)$. However $\bar{H}_{\alpha}=\mathrm{D}_{18}$ is not the centraliser of any element in $\bar{H}_{\alpha}=\operatorname{PSL}(2,8)$, which is not possible.

Thus, $\Gamma$ and $\Gamma_{C}$ are of valency $p^{d}$, and $\bar{G} \cong H_{v}^{\Gamma(v)}=\mathbb{Z}_{p}^{d}: H_{o} \cong \mathbb{Z}_{p}^{d}: \mathbb{Z}_{b}$ is a Frobenius group; in particular, $\bar{G}$ is center free. Hence $\bar{H} \cong\left(\mathbb{Z}_{p}^{d}: H_{o}\right) \times\left(\mathbb{Z}_{p}^{d}: H_{o}\right)$. Now $\Gamma_{C}$ is a holomorph Cayley graph of $\bar{G}=\mathbb{Z}_{p}^{d}: H_{o}$, that is, $\Gamma_{C}=\operatorname{Cay}(\bar{G}, S)$ such that $S$ is a full conjugacy class of elements of $\bar{G}$, and $|S|=p^{d}$. Let $\alpha$ to be the vertex of $\Gamma_{C}$ corresponding to the identity of $\bar{G}$, and let $\beta \in \Gamma_{C}(\alpha)=S$. Then $\bar{H}_{\alpha} \cong \mathbb{Z}_{p}^{d}: H_{o}$, and $\bar{H}_{\alpha \beta} \cong H_{o}$. Since $\Gamma_{C}$ is undirected, we have $S=S^{-1}$ and so $\beta^{-1} \in S$. Now $\mathbf{C}_{\bar{G}}(\beta) \cong \bar{H}_{\alpha \beta}$ so $\beta$ is not order $p$. Further as $\bar{G}=\mathbb{Z}_{p}^{d}: \mathbb{Z}_{b}$ is a Frobenius group, $\beta$ is not conjugate to $\beta^{-1}$ if $o(\beta)>2$. Hence $\beta$ is an involution. It follows that $p$ is odd and $H_{o}=\langle\beta\rangle \cong \mathbb{Z}_{2}$. So $\Gamma_{C} \cong \mathbf{K}_{p^{d}, p^{d}}$, and $\Gamma$ is a normal cover of $\Gamma_{C}$. By Lemma 7 , the theorem holds. 


\section{Subnormal transitive subgroups}

Let $G \triangleleft \triangleleft X \leqslant \operatorname{Sym}(\Omega)$ be such that $G$ is transitive on $\Omega$. Assume that $G=N_{0} \triangleleft N_{1} \triangleleft$ $\cdots \triangleleft N_{r}=X$, where $N_{i+1}=\mathbf{N}_{X}\left(N_{i}\right)>N_{i}$. A natural question is whether $r$ has an upper-bound. For characteristic simple groups, we have a positive answer.

Lemma 11. Let $G \leqslant \operatorname{Sym}(\Omega)$ be a finite characteristic simple group. If $G \triangleleft \triangleleft X \leqslant$ $\operatorname{Sym}(G)$ and $G$ is transitive, then either $G \triangleleft X$, or there exists a group $N$ such that $G \triangleleft N \triangleleft X$.

Proof. Write $G=T^{k}$, where $T$ is a simple group and $k \geqslant 1$. Suppose that $G \triangleleft \triangleleft X \leqslant$ $\operatorname{Sym}(G)$ and $G$ is not normal in $X$. Let $N=\mathbf{N}_{X}(G)$. Then $N<X$, and there exists $x \in X \backslash N$ such that $N^{x}=N$ and $G^{x} \neq G$. Let $C=G \cap G^{x}$ and $H=G G^{x}$. Then $C, G$, and $G^{x}$ are normal in $N$, in particular, $G$ and $G^{x}$ normalizes each other. If $G$ is abelian, then $G$ is regular and $G / C$ is abelian, which is a contradiction to Lemma 10 since now $G^{x} \neq G$. Thus $G$ and so $T$ is nonabelian.

Let $G=N_{0} \triangleleft N_{1} \triangleleft N_{2} \triangleleft \cdots \triangleleft N_{r}=X$. Let $M_{i}=\left\langle G^{x} \mid x \in N_{i}\right\rangle$, where $2 \leqslant i \leqslant r$. We claim that $M_{i}=G \times T^{m_{i}}$ for some positive integer $m_{i}$. First, $M_{2}=\left\langle G^{x} \mid x \in N_{2}\right\rangle \triangleleft N_{2}$. Since $G^{x} \triangleleft N_{1}$ for $x \in N_{2}$, we conclude that $G G^{x}=T^{n}$ for some $n>k$, and as $G \triangleleft G G^{x}$, we have $G G^{x}=G \times T^{l}$. It follows that $M_{2}=G \times T^{m_{2}}$ for some positive integer $m_{2}$. Assume inductively that $M_{i}=G \times T^{m_{i}}$ for some positive integer $m_{i}$. Then $M_{i}=T^{k+m_{i}}$ is a characteristically simple group. Arguing as for $M_{2}$, with $M_{i}$ in the position of $G$, we obtain $M_{i+1}=\left\langle M_{i}^{x} \mid x \in N_{i+1}\right\rangle=M_{i} \times T^{n}=G \times T^{m_{i+1}}$, where $m_{i+1}$ is a positive integer. By induction, $M_{r}=G \times T^{m_{r}}$, and hence $G \triangleleft M_{r} \triangleleft N_{r}=X$.

However, we have been unable to extend this lemma for general groups.

Question 12. Let $G=N_{0} \triangleleft N_{1} \triangleleft \cdots \triangleleft N_{r}=X \leqslant \operatorname{Sym}(\Omega)$, where $N_{i+1}=\mathbf{N}_{X}\left(N_{i}\right)>N_{i}$. Assume that $G$ is transitive. Is it true that $r \leqslant 2$ ?

In the rest of this section, we construct a family of half-symmetric graphs which are subnormal Cayley graphs, and prove Theorem 2.

Let $T$ be a nonabelian simple group, and let $k \geqslant 2$. Let

$$
X=T^{k} \cdot\left(\operatorname{Out}(T) \times \S_{k}\right)
$$

be a primitive permutation group on $\Omega \equiv T^{k-1}$ of simple diagonal type, see [13]. Then the stabilizer

$$
X_{\omega}=D \cdot\left(\operatorname{Out}(T) \times \S_{k}\right)=D . \operatorname{Out}(T) \times \S_{k},
$$

where $D$.Out $(T)=\{(t, t, \ldots, t) \mid t \in \operatorname{Aut}(T)\}$, and the socle $M:=\operatorname{soc}(X)=T^{k}=$ $T_{1} \times T_{2} \times \cdots \times T_{k}$. Let $G \times\{1\}=T_{1} \times \cdots \times T_{k-1} \times\{1\} \triangleleft \operatorname{soc}(X)$, and $N=\mathbf{N}_{X}(G \times\{1\})$. Then $G \times\{1\}$ is regular on $\Omega$, and $N=T^{k}$.(Out $\left.(T) \times \S_{k-1}\right)$.

Proof of Theorem 2: Using the notation defined above, assume further that $k \geqslant 3$, and there exists an element $t \in T$ such that $t$ is not conjugate in $\operatorname{Aut}(T)$ to $t^{-1}$. Let $g=(t, 1, \ldots, 1,1) \in G \times\{1\}$ where $t \in T$, and let

$$
\Gamma=\operatorname{Cos}\left(X, X_{\omega}, X_{\omega}\left\{g, g^{-1}\right\} X_{\omega}\right) .
$$


Then $\Gamma$ is $X$-edge transitive, and $X \leqslant \operatorname{Aut} \Gamma \leqslant \operatorname{Sym}(\Omega)$. Further, $\Gamma$ is not a complete graph, and so Aut $\Gamma \neq \operatorname{Sym}(\Omega)$. By [13], Aut $\Gamma=X$.

Suppose that $\Gamma$ is arc-transitive. Then by Lemma $4, X_{\omega} g X_{\omega}=X_{\omega} g^{-1} X_{\omega}$, and so $g=x g^{-1} y$, for some elements $x, y \in X_{\omega}$. Since $X_{\omega}=D$.Out $(T) \times \S_{k}$, the elements $x=\left(t_{1}, t_{1}, \ldots, t_{1}\right) \pi_{1}$, and $y=\left(t_{2}, t_{2}, \ldots, t_{2}\right) \pi_{2}$, where $t_{i} \in \operatorname{Aut}(T)$, and $\pi_{i} \in \S_{k}$. Thus

$$
(t, 1, \ldots, 1)=g=x g^{-1} y=\left(t_{1}, t_{1}, \ldots, t_{1}\right) \cdot \pi_{1}\left(t^{-1} t_{2}, t_{2}, \ldots, t_{2}\right) \pi_{1}^{-1} \cdot \pi_{1} \pi_{2} .
$$

It follows that $\pi_{1} \pi_{2}=1$, and the element on the right hand side has exactly one entry equal to $t_{1} t^{-1} t_{2}$ and the other entries equal to $t_{1} t_{2}$. Since $k \geqslant 3$, we conclude that $t_{1} t_{2}=1$ and $t=t_{1} t^{-1} t_{2}$. Thus $t=t_{2}^{-1} t^{-1} t_{2}$ and so $t$ is conjugate to $t^{-1}$ which is a contradiction. Hence $\Gamma$ is half-symmetric.

Finally, since $G \times\{1\}$ is regular on $\Omega, \Gamma$ is a Cayley graph of $G \times\{1\}$, that is, $\Gamma=\operatorname{Cay}(G \times\{1\}, S \times\{1\})$ for some subset $S \times\{1\} \subset G \times\{1\}$. Let $\omega$ be the vertex corresponding to $X_{\omega}$, let $\beta=X_{\omega} g$. Then the stabilizer of $\omega$ in $X$ is $X_{\omega}$, and the stabilizer of $\beta=X_{\omega}^{g}$. So $X_{\omega \beta}=X_{\omega} \cap X_{\omega}^{g}=\mathbf{C}_{\text {Aut }(T)}(g) \times \S_{k-1}$. Since $g$ is not conjugate to $g^{-1}$, we have $\Gamma$ is not $X$-arc transitive. By Lemma 2.1 in [12], $X_{\omega}$ have two orbits of the same size on $\Gamma(\omega)$, and each have size $\operatorname{val}(\Gamma)=\left|X_{\omega}: X_{\omega \beta}\right|=\left|\operatorname{Aut}(T): \mathbf{C}_{\text {Aut }(T)}(g)\right| . k$. Thus

$$
\operatorname{val}(\Gamma)=2\left|X_{\omega}: X_{\omega \beta}\right|=2\left|\operatorname{Aut}(T): \mathbf{C}_{\text {Aut }(T)}(g)\right| . k .
$$

Let $\pi=(12 \ldots k)$, and let $g_{i}=g^{\pi^{i}}$. Then the $i$-th entry of $g_{i}$ is $t$ and the others equal 1 , and

$$
X_{\omega}\left\{g, g^{-1}\right\} X_{\omega}=\left\{X_{\omega} g_{i}^{x}, X_{\omega}\left(g_{i}^{-1}\right)^{x} \mid x \in X_{\omega}\right\} .
$$

Note that $g_{i}^{x}=\left(1, \ldots, t^{x_{i}}, \ldots, 1\right)$, where $x_{i} \in \operatorname{Aut}\left(T_{i}\right)$. For $i \leqslant l=k-1$, let $\bar{g}_{i}^{x}$ be the projection of $g_{i}^{x}$ in $G=T_{1} \times \cdots \times T_{k-1}$. For $i=k, g_{k}^{x}$ has the following property

$$
g_{k}^{x}=\left(1, \ldots, 1, t^{x_{k}}\right) \equiv\left(\left(t^{x_{k}}\right)^{-1}, \ldots,\left(t^{x_{k}}\right)^{-1}, 1\right)\left(\bmod X_{\omega}\right) .
$$

Let $\bar{g}_{k}^{x}=\left(\left(t^{x_{k}}\right)^{-1}, \ldots,\left(t^{x_{k}}\right)^{-1}\right)$ be the projection of $g_{k}^{x}$ in $G$. Then all $\bar{g}_{i}^{x}$ for $1 \leqslant i \leqslant k$ lie in $S$. Similarly, we have the projections $\left(\bar{g}_{i}^{-1}\right)^{x}$ of $\left(g_{i}^{-1}\right)^{x}$. Since $|S|=|S \times\{1\}|=$ $\operatorname{val}(\Gamma)=2\left|\operatorname{Aut}(T): \mathbf{C}_{\text {Aut }(T)}(g)\right| . k$, it follows that

$$
S=\left\{\bar{g}_{i}^{x},\left(\bar{g}_{i}^{-1}\right)^{x} \mid 1 \leqslant i \leqslant k, x \in X_{\omega}\right\} .
$$

Thus $\Gamma$ can be represented as a Cayley graph of $G$, that is, $\Gamma \cong \operatorname{Cay}(G, S) \cong \operatorname{Cay}(G \times$ $\{1\}, S \times\{1\})$. As $G \cong G \times\{1\} \triangleleft \operatorname{soc}(X) \triangleleft X$, the Cayley graph $\Gamma$ is subnormal and has the form stated in Theorem 2 .

\section{Acknowledgements}

The author want to thank professor Shaofei Du for giving me valuable suggestions, thank professor Qing Xiang for suggesting the proof of Lemma 8. 


\section{References}

[1] N. Biggs, Algebraic Graph Theory, Second edition. Cambridge Mathematical Library. Cambridge University Press, Cambridge, 1993. viii+205 pp. ISBN: 0-521-45897-8

[2] P.J. Cameron, Permutation groups, Cambridge University Press, Cambridge, 1999.

[3] J. D. Dixon and B. Mortimer, Permutation Groups, Springer, New York, Berlin (1996).

[4] X. G. Fang, C. H. Li and C. E. Praeger, The locally 2-arc-transitive graphs admitting a Ree simple group, J. Algebra 282 (2004), 638-666.

[5] X. G. Fang, C. H. Li, J. Wang and M. Y. Xu, On cubic normal Cayley graphs of finite simple groups, Discrete Math. 244 (2002), 67-75.

[6] Yan-Quan Feng and Ming-Yao Xu, Normality of tetravalent Cayley graphs of odd prime-cube order and its application, Acta Mathmatica Sinica, English Series,21 (2005), 903-912.

[7] C. D. Godsil, On the full automorphism group of a graph, Combinatorica 1 (1981), 243-256.

[8] C.Hering, Transitive linear groups and linear groups which contain irreducible sugroups of prime order. Germetriae Dedicata, 2 (1974), 425-460.

[9] C. H. Li, On finite groups with the Cayley invariant property, Bull. Austral. Math. Soc. 56 (1997), 253-261.

[10] C. H. Li, Finite $s$-arc transitive Cayley graphs and flag-transitive projective planes, Proc. Amer. Math. Soc. 133 (2004), 31-41.

[11] C. H. Li, Finite edge-transitive Cayley graphs and rotary Cayley maps, Trans. Amer. Math. Soc. 358 (2006), 4605-4635.

[12] C. H. Li, J. Pan, S. J. Song, D. Wang, A characterisation of a family of edge-transitive metacirculant graphs, J. Comb. Theory, Series B, 107 (2014), 12-25.

[13] M. Liebeck, C. E. Praeger and J. Saxl, A classification of the maximal subgroups of the finite alternating and symmetric groups, J. Algebra 111 (1987), 365-383.

[14] D. Marušič, Quartic half-arc-transitive graphs with large vertex stabilizers, Discrete Math. 299 (2005), no. 1-3, 180-193.

[15] C. E. Praeger, An O'Nan-Scott theorem for finite quasiprimitive permutation groups and an application to 2-arc transitive graphs, J. London. Math. Soc. 47 (1992), 227239.

[16] C. E. Praeger, Finite normal edge-transitive Cayley graphs, Bull. Austral. Math. Soc. 60 (1999), 207-220.

[17] S. J. Song, C. H. Li, H. Zhang, Finite permutation groups with a regular dihedral subgroup, and edge-transitive dihedrants, J. Algebra, 399 (2014), 948-959.

[18] H. Wielandt, Finite Permutation Groups, Academic Press, New York, London (1964).

[19] M. Y. Xu, Automorphism groups and isomorphisms of Cayley digraphs, Discrete Math. 182 (1998), 309-320. 